Document downloaded from:

http://hdl.handle.net/10251/61116

This paper must be cited as:

Vayá Pérez, I.; Bonancía Roca, P.; Jiménez Molero, MC.; Markovitsi, D.; Gustavsson, T.; Miranda Alonso, MÁ. (2013). Excited state interactions between flurbiprofen and tryptophan in drug-protein complexes and in model dyads. Fluorescence studies from the femtosecond to the nanosecond time domains. Physical Chemistry Chemical Physics. 15(13):4727-4734. doi:10.1039/c3cp43847c.

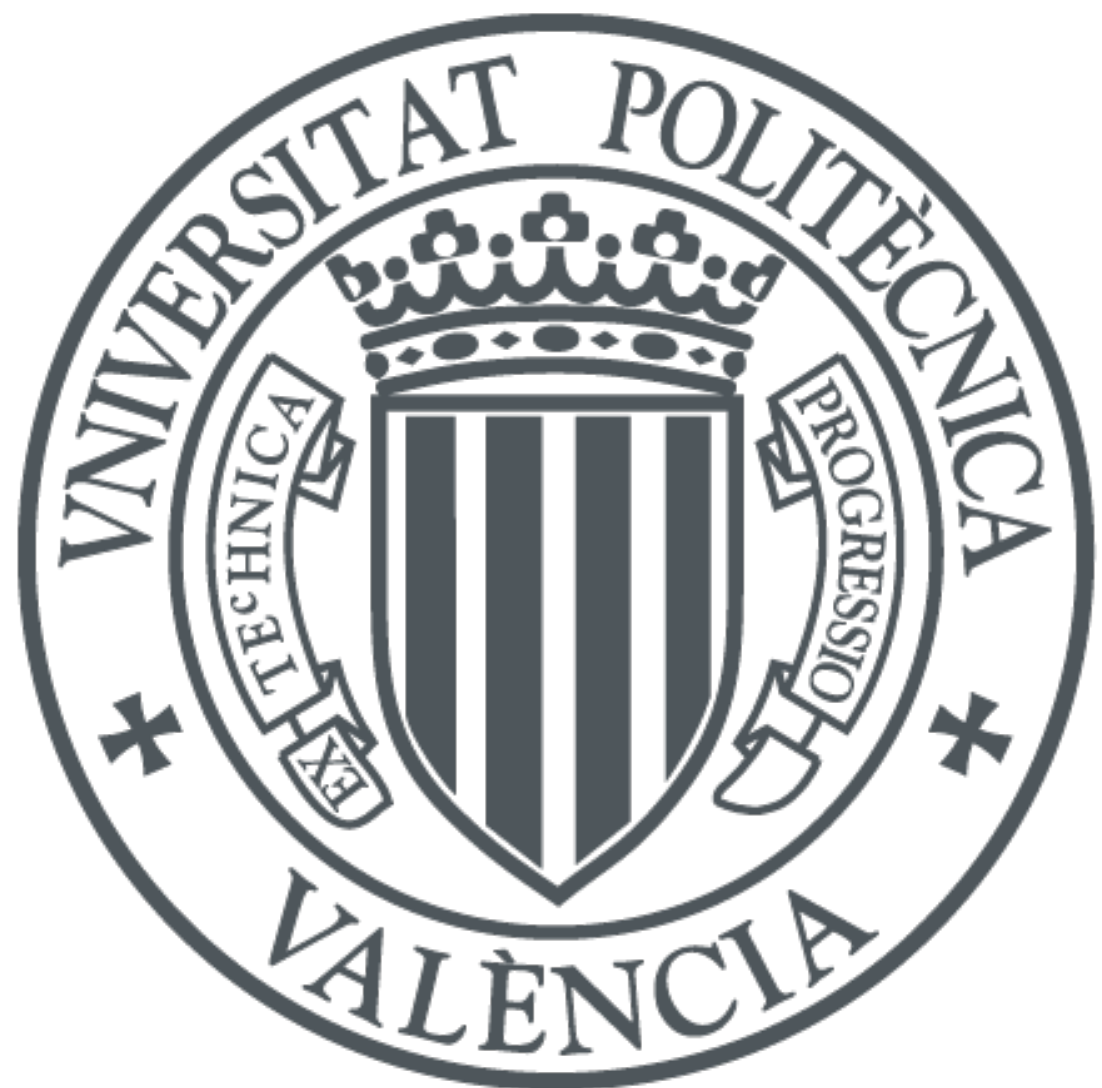

The final publication is available at

http://dx.doi.org/10.1039/c3cp43847c

Copyright Royal Society of Chemistry

Additional Information 


\title{
Excited state interactions between flurbiprofen and tryptophan in drug/protein complexes and in model dyads. Fluorescence studies from the femtosecond to the nanosecond time domains
}

\author{
Ignacio Vayá, ${ }^{a}$ Paula Bonancía, ${ }^{a}$ M. Consuelo Jiménez, ${ }^{a}$ Dimitra Markovitsi, ${ }^{b}$ Thomas Gustavsson ${ }^{b} *$ and \\ ${ }_{5}$ Miguel A. Miranda ${ }^{a}$ *
}

We report here on the interaction dynamics between flurbiprofen (FBP) and tryptophan (Trp) covalently linked in model dyads and in a complex of FBP with human serum albumin (HSA) probed by time-

10 resolved fluorescence spectroscopy from the femto- to the nano-second timescales. In the dyads, a rapid $\left(\mathrm{k}>10^{10} \mathrm{~s}^{-1}\right)$ dynamic quenching of the ${ }^{1} \mathrm{FBP} *$ fluorescence is followed by a slower $\left(\mathrm{k}>10^{9} \mathrm{~s}^{-1}\right)$ quenching of the remaining ${ }^{1} \operatorname{Trp} *$ fluorescence. Both processes display a clear stereoselectivity; the rates are 2-3 times higher for the $(R, S)$-dyad. In addition, a red-shifted exciplex emission is observed, rising in 100-200 ps. A similar two-step dynamic fluorescence quenching is also observed in the FBP/HSA

15 complex, although the kinetics of the involved processes are slower. The characteristic reorientational times determined for the two enantiomeric forms of FBP in the protein show that the interaction is stronger for the $(R)$ - form. This is, to our knowledge, the first observation of stereo-selective flurbiprofentryptophan interaction dynamics with femtosecond time resolution.

\section{Introduction}

20 The binding of drugs to biomolecules is determinant not only for drug action (both therapeutic and toxic) but also for drug transport and disposition, which are regulated by various transport proteins such as human serum albumin (HSA). The detailed understanding of drug-protein binding, both from a 25 structural and dynamic point of view, constitutes a particularly active research field today.

Actually, HSA is one of the most abundant proteins in blood and plasma and is responsible for the transport of different agents in the bloodstream, such as fatty acids, drugs, or metabolites. ${ }^{1,2}$ 30 Therefore, the binding of ligands to HSA constitutes a key process, relevant for the modulation of a number of properties (drug solubility in plasma, toxicity, susceptibility to oxidation, in vivo half-life, etc.). ${ }^{3,4}$

Flurbiprofen (FBP, Chart 1) [2-(2-fluorobiphenyl-4$35 \mathrm{yl}$ )propanoic acid] is a nonsteroidal anti-inflammatory drug (NSAID) employed for the treatment of a broad spectrum of pathophysiological conditions, including fever, headache, etc. ${ }^{5-8}$ Moreover, FBP presents a chiral centre, and it has been reported that mainly the $(S)$-form possesses pharmacological activity 40 (cyclooxygenase inhibition). ${ }^{9,10}$ It is known that FBP binds to HSA preferentially in the so-called site II following Sudlow's classification. $^{11}$

Optical spectroscopy has proven to be particularly useful in the study of drug-protein binding. ${ }^{12,13}$ The observed excited state 45 dynamics may be interpreted in terms of fundamental processes such as energy and charge transfer, depending on the specific drug-protein binding. Characterisation of the excited states provides a better understanding of the molecular recognition governing the drug transport. In particular, different enantiomers

50 may have different binding behaviour resulting in distinct spectroscopic properties.

From the photophysical point of view, FBP contains a biphenyl chromophore with well-known properties. ${ }^{14}$ It exhibits a non-negligible intersystem crossing yield, so both the singlet and 55 triplet states can in principle serve to monitor its binding to a protein. Indeed, previous laser flash photolysis studies on FBP/HSA complexes showed that FBP binds to both sites I and II, but with higher affinity to site II. ${ }^{15}$ The triplet lifetime of FBP within the protein is similar for the two enantiomers; however, 60 this parameter monitors triplet state protection from oxygen quenching, rather than direct interaction with the protein. In fact, the fundamental processes involved in the binding dynamics occur on a much shorter time scale, a few nanoseconds or less.

Fluorescence spectroscopy provides therefore a more direct 65 means to study the early events of molecular recognition since it involves the singlet excited state of the drug and its dynamics, which in most cases evolves on the nanosecond timescale. Various fluorescence techniques have been used in the past to investigate the interactions between different drugs and proteins, 70 with special attention to HSA. In addition to steady-state fluorescence quenching, ${ }^{16-24}$ time-resolved measurements ${ }^{25-27}$ allow the characterisation of the involved dynamic processes. In particular, femtosecond emission ${ }^{28-32}$ constitutes a powerful tool for determining the very fast photo-initiated processes.

75 Steady-state fluorescence titration and anisotropy 
measurements have been used to probe the FBP/HSA complex. ${ }^{11,14,33,34}$ Time-resolved techniques have been applied to characterise the singlet excited states of both $\mathrm{FBP}^{14}$ and HSA, ${ }^{36}$ but not on the FBP/HSA complex, probably because the 5 absorption spectra of the two compounds overlap strongly, and their selective excitation is not possible.

It is well established that the UVB-induced fluorescence of HSA is mainly due to Trp- $214,{ }^{36}$ which can in principle facilitate the discrimination between FBP and HSA fluorescence, needed 10 in order to evaluate the individual quenching rates. It is

worthwhile to note the high sensitivity of Trp emission to its local microenvironment. Thus, spectral changes can be observed in response to protein conformational transitions, ligand binding or subunit association. Moreover, Trp is sensitive to collisional 15 quenching, probably due to the capability of the excited-state of indole to act as an electron donor. ${ }^{36}$

In view of the complexity of the FBP/HSA system and the potential difficulties to interpret the fluorescence properties, complementary information is necessary in order to investigate 20 the specific interactions between FBP and Trp. To this purpose, covalently linked dyads formed by FBP and $(S)$-TrpMe $((S)$ tryptophan methyl ester) have been employed as simple models for investigation of the key phenomena occurring in drug-protein interactions. $^{37}$

25 Related drug-amino acid dyads have already been designed and studied with success in the past. ${ }^{21,37-40}$ They have provided new mechanistic insight into the key processes that occur between the two chromophores (such as energy transfer, electron transfer, exciplex formation, etc.). Interestingly, the picture 30 obtained regarding the covalently linked dyads can be usually extended to the more complex drug-protein systems.

Spectroscopic studies on FBP-TrpMe dyads revealed the absence of any significant ground-state intramolecular interactions between the two chromophores. ${ }^{37}$ Fluorescence 35 spectra recorded at $\lambda_{\text {exc }}=266 \mathrm{~nm}$ (where $c a$. $60 \%$ of the light is absorbed by the biphenyl and $40 \%$ by the indole chromophore) displayed a dramatic FBP fluorescence quenching and a residual emission $\left(\lambda_{\max }=340 \mathrm{~nm}\right)$ assigned to the TrpMe unit. This highly efficient FBP fluorescence quenching was explained by energy ${ }_{40}$ transfer from ${ }^{1} \mathrm{FBP} *$ to TrpMe, ${ }^{37}$ which is in accordance with the excited state energy of FBP ( $99 \mathrm{kcal} \mathrm{mol}^{-1}$ ), higher than that of Trp $\left(96 \mathrm{kcal} \mathrm{mol}^{-1}\right){ }^{14,41}$ Moreover, the non-negligible spectral overlap between FBP emission and TrpMe absorption spectra would be in favour of Förster energy transfer. Concerning the

45 nanosecond time-resolved measurements, the fluorescence lifetimes at $\lambda_{\mathrm{em}}=340 \mathrm{~nm}$ were much shorter in the dyads $\left(\tau_{\mathrm{F}}<\right.$ $1 \mathrm{~ns})$ than in $(S)$-TrpMe, indicating a dynamic quenching. However, these $\tau_{\mathrm{F}}$ values were judged inaccurate, due to the limitations of the equipment. This quenching was assigned to 50 either electron transfer or exciplex formation. Both processes are thermodynamically allowed, according to the Rehm-Weller equations. ${ }^{42}$ Exciplex emission was indeed detected as a broad band between $380 \mathrm{~nm}$ and $500 \mathrm{~nm}$, especially in $(R, S)$-FBPTrpMe.

55 With this background, it appeared interesting to reinvestigate the singlet excited state interactions occurring in both FBP/HSA complexes and FBP-TrpMe model dyads (Scheme 1), using fluorescence techniques with a much higher time-resolution.
In order to overcome the abovementioned spectral overlap 60 issue, the fluorescence decays were monitored at chosen wavelengths where the emission is dominated by FBP $(310 \mathrm{~nm})$ or $\operatorname{Trp}(340-380 \mathrm{~nm})$.

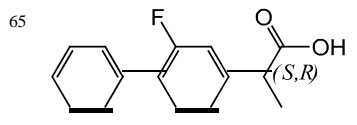

FBP

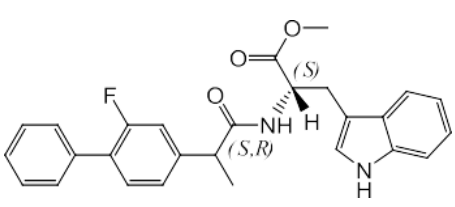

FBP-TrpMe
Scheme 1

\section{Experimental}

The $(S)$ - and $(R)$-enantiomers of flurbiprofen $((S)$ - and $(R)$ FBP), $(S)$-tryptophan methyl ester $((S)$-TrpMe) and human serum albumin (HSA) were purchased from Sigma-Aldrich. Acetonitrile 75 was of HPLC quality from Merck. The PBS buffer was prepared by dissolving phosphate-buffered saline tablets (Sigma) in ultrapure water from a Millipore (Milli-Q Synthesis) system. The synthesis of the FBP-TrpMe model dyads is already reported. ${ }^{37}$

Steady-state absorption spectra were recorded with a Perkin${ }_{80}$ Elmer Lambda 900 spectrophotometer. Steady-state fluorescence spectra were obtained using a SPEX Fluorolog-3 spectrofluorometer, with an excitation wavelength of $267 \mathrm{~nm}$ at $22{ }^{\circ} \mathrm{C}$. Solutions were placed into $10 \mathrm{~mm} \times 10 \mathrm{~mm}$ quartz cells. The absorbance of the samples at the excitation wavelength was ${ }_{85}$ kept below 0.2. Fluorescence quantum yields were determined using FBP in MeÇN/air as a secondary reference, with $\phi_{\mathrm{F}}=0.17$ (air) or $0.21\left(\mathrm{~N}_{2}\right)$.

Time-resolved fluorescence measurements were performed using the fluorescence upconversion (FU) and time-correlated 90 single photon counting (TCSPC) techniques. The excitation source was the third harmonic $(267 \mathrm{~nm})$ of a mode-locked TiSapphire laser, delivering $\sim 120$ fs pulses whose repetition rate was 76 and $4.75 \mathrm{MHz}$ for FU and TCSPC, respectively (in the latter case set by a pulse-picker).

95 For the FU measurements, a home-built setup was used. This has been described in detail earlier. Briefly, a $1 \mathrm{~mm}$ type I BBO sum-frequency crystal was used, providing an instrumental response function of about $350 \mathrm{fs}$ (fwhm). We judge that the time resolution of the setup is better than $100 \mathrm{fs}$ after deconvolution,

100 depending on the signal-to-noise ratio. Typical scans were performed in a 200 picosecond time interval with a 1 ps step. The average excitation power used was $40 \mathrm{~mW}$. The power density cannot be measured precisely within the excitation volume but we estimate it to $0.2 \pm 0.1 \mathrm{GW} / \mathrm{cm}^{2}$ for a $40 \mathrm{~mW}$ output from the 105 tripler unit (assuming a 40 micron diameter of the focused beam). Solutions (about $25 \mathrm{ml}$ ) were kept flowing through a $0.4 \mathrm{~mm}$ quartz cell, which was kept in continuous motion perpendicular to the excitation beam in order to minimise thermal effects.

For the TCSPC experiments, a Becker \& Hickl GmbH PC card 110 was used. ${ }^{45}$ A Schott WG 295 filter was placed in front of a SPEX monochromator. The detector was a microchannel plate (R1564 U Hamamatsu) providing an instrumental response function of $60 \mathrm{ps}$ (fwhm). The average laser power $(0.1 \mathrm{~mW})$ was measured with a Melles Griot broadband powermeter. The 115 irradiated area on the surface of the cell was ca. $0.2 \mathrm{~cm}^{2}$ 
corresponding to a pulse intensity of $2.4 \mathrm{~kW} / \mathrm{cm}^{2}$. Solutions were contained in a $10 \mathrm{~mm} \times 10 \mathrm{~mm}$ quartz cell and continuously stirred. Successive recordings with the same sample gave identical decays, which were eventually merged to improve the 5 signal-to-noise ratio. Such a procedure allowed us to ensure that the measured signals were not altered during the measurements due to a possible accumulation of photoproducts.

The time-resolved experiments were performed either at magic angle or under successive parallel $\left(I_{p a r}(t)\right)$ and perpendicular ${ }_{10}\left(I_{\text {perp }}(t)\right)$ excitation/detection conditions. These were achieved by controlling the polarisation of the exciting beam with a zero-order half-wave plate. From these measurements, the fluorescence anisotropy was calculated from the formula

15

$$
\text { ( ) } \frac{() \quad()}{()}
$$

The transmission under parallel and perpendicular conditions was found to be identical so the correction factor $R$ was put to unity.

\section{Results and discussion}

\section{${ }_{20} 3.1$ Studies on FBP-TrpMe model dyads}

Due to the poor solubility of the FBP-TrpMe dyads in aqueous media, experiments were performed in acetonitrile, under aerated conditions. Steady-state absorption and fluorescence spectra of $(S)$-FBP, $(S)$-TrpMe, $(S, S)$-FBP-TrpMe and $(R, S)$-FBP-TrpMe in 25 acetonitrile/air are given in Figures SI-1 and SI-2. As previously described, a dramatic fluorescence quenching (>90\%) was observed for the dyads. The fluorescence quantum yields of $(S, S)$ and $(R, S)$-FBP-TrpMe were found to be 0.028 and 0.015 , respectively (Figure SI-2A), which are much lower than those of 30 the individual chromophores. ${ }^{38}$ From the shape and the position of the fluorescence bands, it was confirmed that emission is dominated by ${ }^{1} \mathrm{TrpMe}{ }^{*}$. For both dyads, a longer wavelength band (centred at $450 \mathrm{~nm}$ ), assigned to exciplex emission, was also observed; it was more intense for the $(R, S)$ - diastereomer (Figure $\left.{ }_{35} \mathrm{SI}-2 \mathrm{~B}\right)$.

Figure 1 shows the fluorescence decays recorded by FU at both 310 and $340 \mathrm{~nm}$ (emission maxima of FBP and TrpMe, respectively). The decay kinetics of the dyads were much faster than those of FBP or TrpMe. This is in line with the relative 40 quantum yields and clearly shows the dynamic nature of the fluorescence quenching.

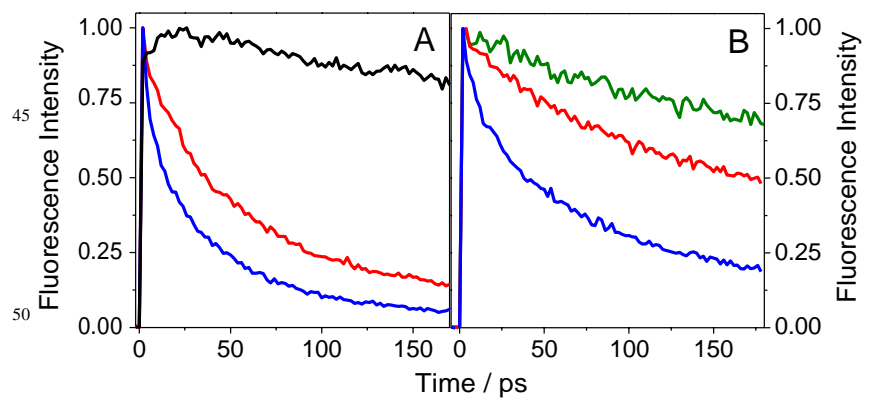

Figure 1. Normalised FU decays at A) $\left(\lambda_{\mathrm{em}}=310 \mathrm{~nm}\right)$ and $\left.\mathrm{B}\right)\left(\lambda_{\mathrm{em}}=340\right.$ $\mathrm{nm})$ of $(S)$-FBP (black), $(S)$-TrpMe (green), $(S, S)$-FBP-TrpMe (red) and $55(R, S)$-FBP-TrpMe (blue).
At $310 \mathrm{~nm}$, where FBP emission is dominating, the most striking feature was the very rapid decay of the dyads, on the picosecond time scale (Figure 1A). In addition, a significant stereo-differentiation was noticed; the $(R, S)$ - dyad emission 60 decayed faster than that of the $(S, S)$-diastereomer. The fluorescence decays were highly non-exponential, but the "average" characteristic times estimated at the 1/e level were 62 and 28 ps for $(S, S)$ - and $(R, S)$-FBP-TrpMe, respectively (Table $1)$. These times should be compared to the much longer and 65 wavelength independent fluorescence lifetime of FBP (1.67 ns). The FBP signal at $310 \mathrm{~nm}$ shows a rapid rise, on the order of a few $\mathrm{ps},{ }^{46}$ which can in principle be assigned to a vibrational redistribution in the excited state.

Based on the average lifetimes values given above, the 70 corresponding rate constants $\left(\mathrm{k}_{\mathrm{Q} 1}\right)$ were estimated (Table 1$)$. They were higher than $10^{10} \mathrm{~s}^{-1}$ and revealed a remarkable stereoselectivity.

Table 1. Kinetic parameters derived from the FU and TCSPC 75 fluorescence decays of $(S)$-FBP, $(S)$-TrpMe, $(S, S)$-FBP-TrpMe and $(R, S)$ FBP-TrpMe in acetonitrile under air at 310 and $340 \mathrm{~nm}$. Uncertainties are $\pm 5 \%$ if not otherwise stated.

\begin{tabular}{ccccc}
\hline Compound & $\tau_{\mathrm{F}}(\mathrm{ps})^{\mathrm{a}}$ & $\mathrm{k}_{\mathrm{Q} 1} \times 10^{10}\left(\mathrm{~s}^{-1}\right)$ & $\tau_{\mathrm{F}}(\mathrm{ns})^{\mathrm{b}, \mathrm{c}}$ & $\mathrm{k}_{\mathrm{Q} 2} \times 10^{9}\left(\mathrm{~s}^{-1}\right)$ \\
\hline$(S)$-FBP & $1670^{\mathrm{c}}$ & - & - & \\
$(S)$-TrpMe & $2100^{\mathrm{c}}$ & - & 1.35 & \\
$(S, S)$-FBP-TrpMe & $62^{\mathrm{d}}$ & 1.6 & 0.46 & 1.4 \\
$(R, S)$-FBP-TrpMe & $28^{\mathrm{d}}$ & 3.5 & 0.23 & 3.6 \\
\hline
\end{tabular}

${ }^{\mathrm{a}} \lambda_{\mathrm{em}}=310 \mathrm{~nm} ;{ }^{\mathrm{b}} \lambda_{\mathrm{em}}=340 \mathrm{~nm} ;{ }^{\mathrm{c}} \mathrm{TCSPC} ;{ }^{\mathrm{d}} \mathrm{FU}$

The FU decays at $340 \mathrm{~nm}$ (Figure 1B) were slower than those 80 observed at $310 \mathrm{~nm}$. Also here, the decays were highly nonexponential with average characteristic times of 300 and $80 \mathrm{ps}$ for $(S, S)$ - and $(R, S)$-FBP-TrpMe, respectively. No rise in the signal, supporting the previous proposed energy transfer from ${ }^{1} \mathrm{FBP} *$ to TrpMe, was observed. However, such a rise could be 85 experimentally difficult to detect, taking into account the strong direct excitation of TrpMe at $267 \mathrm{~nm}$ and the spectral overlap of FBP and TrpMe emission at this wavelength.

The fluorescence anisotropy decays of the dyads were compared to that of FBP at $310 \mathrm{~nm}$ (Figure SI-3A). For the latter, 90 the anisotropy decayed with a characteristic time of about $26 \pm 1$ ps, while for $(S, S)$ - and $(R, S)$-FBP-TrpMe characteristic times of $42 \pm 2$ and $44 \pm 4$ ps were obtained. The FBP molecular volume of FBP is $c a .300 \AA^{3}$, which in the frame of the Stokes-EinsteinDebye theory ${ }^{46}$ corresponds to a rotational time of about $27 \mathrm{ps}$, in 95 correspondence with that observed. The total volume of the FBPTrpMe dyads is about $492 \AA^{3}$, giving a rotational time of about $44 \mathrm{ps}$, once again in agreement with those experimentally observed. The mono-exponential behaviour of the anisotropy decays, and in particular the lack of any fast decays at early 100 times, shows that there is no internal rotation between the two chromophores at early times. The slight difference between the $(S, S)$ - and $(R, S)$ - dyads is within the experimental uncertainties.

The fluorescence anisotropy decays of the dyads were also compared to that of TrpMe at $340 \mathrm{~nm}$ (Figure SI-3B). The 105 characteristic times were $24 \pm 1$ and $32 \pm 2$ ps for $(S, S)$ - and $(R, S)$ FBP-TrpMe respectively, faster than to those recorded at $310 \mathrm{~nm}$. This is not necessarily indicative of any internal dynamics, but may only be the result of the disappearance of FBP (higher anisotropy, $r_{0}=0.31 \pm 0.01$ ) leaving only TrpMe (lower 
anisotropy, $\left.r_{0}=0.17 \pm 0.01\right)$ at this wavelength. The zero time fluorescence anisotropies for $(S, S)$ - and $(R, S)$-FBP-TrpMe at 340 $\mathrm{nm}$ are $0.25 \pm 0.01$ and $0.22 \pm 0.01$, respectively, representing average values of FBP and TrpMe. As in the case of the 5 fluorescence intensity decays, these observations can be explained in terms of the direct excitation of TrpMe and the spectral overlap of the two chromophores at $340 \mathrm{~nm}$.

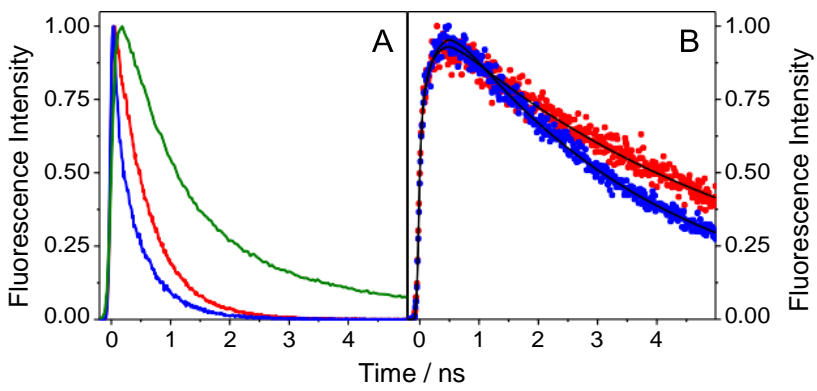

Figure 2. Normalised TCSPC decay traces at A) $\left.\left(\lambda_{\mathrm{em}}=340 \mathrm{~nm}\right), \mathrm{B}\right)\left(\lambda_{\mathrm{em}}\right.$ $=450 \mathrm{~nm})$ of $(S)$-TrpMe (green), $(S, S)$-FBP-TrpMe (red) and $(R, S)$-FBP10 TrpMe (blue). Fitted curves are shown in black.

In order to follow the dynamics at $340 \mathrm{~nm}$, it is necessary to go to longer timescales than with FU. This was achieved by means of TCSPC recorded at $340 \mathrm{~nm}$ (Figure 2A). The dyad 15 signals decayed much faster than that of TrpMe, pointing to a dynamic quenching occurring on a much slower timescale than that observed by means of FU.

What is striking is the clear difference observed in the decay traces of the $(S, S)$ - and $(R, S)$ - dyads. This stereo-differentiation 20 was not detected in the previous study, ${ }^{37}$ because of insufficient time-resolution, and constitutes an important new element. As for FU data, $(R, S)$-FBP-TrpMe decayed more rapidly than $(S, S)$-FBPTrpMe. Both signals were close to mono-exponential, with characteristic times of 460 and $230 \mathrm{ps}$ for the $(S, S)$ - and $(R, S)$ -

${ }_{25}$ diastereomers, respectively (see Table 1 ). These times should be compared to the $1.35 \mathrm{~ns}$ of $\operatorname{TrpMe}$ at this wavelength. The $\mathrm{k}_{\mathrm{Q} 2}$ values of the dyads, calculated from the corresponding $\tau_{\mathrm{F}}$ values, underline the strong stereoselectivity.

The TCSPC decays of the dyads were much faster at $310 \mathrm{~nm}$ 30 than at $340 \mathrm{~nm}$ (Figure SI-4). This should not be surprising in view of the efficient FBP fluorescence quenching. In fact, emission from FBP was not expected beyond a few tens of picoseconds, so the residual fluorescence should be ascribed to the TrpMe unit at both wavelengths. Still, the fluorescence 35 lifetimes measured at 310 and $340 \mathrm{~nm}$ were very different. Actually, the Trp fluorescence is known to be very complex, with strongly wavelength dependent decay times. ${ }^{36}$ This has been assigned to the co-existence of several rotamers with different excited state dynamics, ${ }^{47}$ which may also occur in the dyads.

${ }_{40}$ The TCSPC profiles at $450 \mathrm{~nm}$ (Figure 2B) were much slower than those at either 310 or $340 \mathrm{~nm}$. Interestingly, these profiles are characterised by a rapid rise, $115 \pm 7$ and $189 \pm 6$ ps for $(S, S)$ and $(R, S)$-, respectively, which can be assigned to the formation of an exciplex. ${ }^{38}$ Its rate is intermediate between the two 45 quenching processes described above, so it is not possible to correlate it with any of them. As already proposed in the literature, such exciplexes could serve as intermediates for full electron transfer processes,${ }^{48}$ consistent with the electron donor character of Trp. ${ }^{36}$ The exciplex lifetimes were $5.34 \pm 0.02$ and ${ }_{50} 3.63 \pm 0.01 \mathrm{~ns}$ for the $(S, S)$ - and $(R, S)$ - diastereomers,

respectively.

\subsection{Studies on FBP/HSA complexes}

Steady-state UV absorption and fluorescence measurements were performed on mixtures of $(S)$-FBP or $(R)$-FBP $\left(2.5 \times 10^{-5}\right.$ $\left.{ }_{55} \mathrm{M}\right)$ and HSA $\left(3.6 \times 10^{-5} \mathrm{M}\right)$ in PBS. As the binding constants of FBP to the binding affinity site of HSA are in the order or $10^{6} \mathrm{M}$ ${ }^{1}$, all the drug is essentially bound to the protein under these conditions. $^{11,15}$

The results for $(S)$-FBP are shown in Figure 3. With the $(R)$ 60 enantiomer, the results were basically identical (data not shown). A significant fluorescence quenching was observed for the complexes, even though less important and less stereoselective than for the dyads. This can be interpreted by weaker interaction in the non-covalent supramolecular complexes compared to the ${ }_{65}$ dyads. Comparison of the fluorescence spectra of the drug/protein mixtures with those of isolated FBP and HSA revealed that both components contribute to the emission spectra. This is in principle not unexpected, since both chromophores absorb strongly at the excitation wavelength.

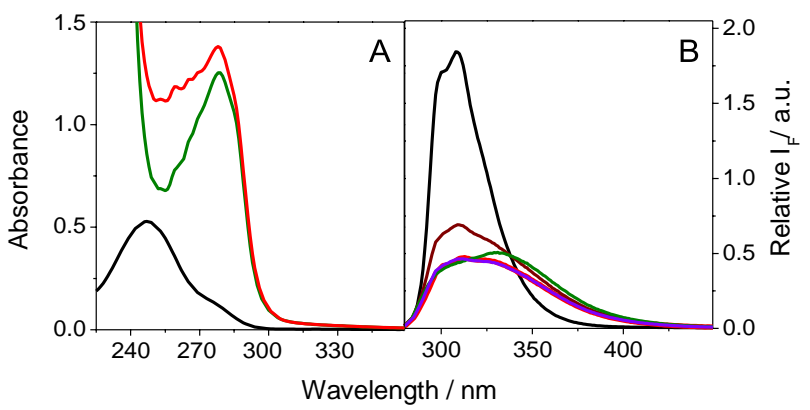

70 Figure 3. A) UV absorption spectra of (S)-FBP (black), HSA (green), and the $(S)$-FBP/HSA mixture (red) in PBS. The concentration of the solutions was $2.5 \times 10^{-5} \mathrm{M}$ for FBP and $3.6 \times 10^{-5} \mathrm{M}$ for HSA; B) fluorescence spectra of (S)-FBP (black), HSA (green), and $(S)$-FBP/HSA (red) in PBS, under air, using isoabsorptive solutions at the excitation

75 wavelength $(267 \mathrm{~nm})$. Simulated emissions, taking into account the percentage of light absorbed by each subunit (dark red), and the possible quenching processes as explained in the text (violet) are also shown.

Taking into account the relative absorbances of FBP and HSA 80 at $267 \mathrm{~nm}$, and assuming independent emission, the fluorescence spectrum of the mixture could in principle be calculated using the simple relation

$$
\mathrm{A}_{\mathrm{F}}(\mathrm{tot})=0.18 \times \mathrm{A}_{\mathrm{F}}(\mathrm{FBP})+0.82 \times \mathrm{A}_{\mathrm{F}}(\mathrm{HSA})
$$

85

where $A_{F}(F B P)$ and $A_{F}(H S A)$ are the areas under the emission curves of the two subunits. However, this simulated spectrum did not match the experimental one. Instead, an excellent reproduction of the real emission of the drug/protein system 90 (Figure 3B) was achieved by using the relation

$$
\mathrm{A}_{\mathrm{F}}(\mathrm{tot})=0.074 \times \mathrm{A}_{\mathrm{F}}(\mathrm{FBP})+0.746 \times \mathrm{A}_{\mathrm{F}}(\mathrm{HSA})
$$


where an important fluorescence quenching of FBP fluorescence and, to a lower extent, of HSA became evident.

In order to gain further insight into the excited state dynamics of these systems, FBP, HSA and the two complexes were 5 investigated by FU and TCSPC at various wavelengths. While the FBP decay in PBS solution was monoexponential, those of HSA and FBP/HSA were strongly non-exponential and wavelength dependent. In general, three-exponential model functions were required for a good fitting of the kinetic traces in the protein10 containing samples (Table 2). In view of the complexity of the fluorescence decay analysis, we also report the average lifetime $(\langle\tau\rangle)$, which allows an easier comparison of the excited state dynamics of FBP in the presence and absence of protein.

The FU measurements of FBP and the FBP/HSA systems 15 recorded at $310 \mathrm{~nm}$ are shown in Figure 4. At early times, the behaviour of the two complexes was identical, showing an instantaneous rise limited by the apparatus function, followed by a constant value (Figure 4A). Interestingly, the signal of FBP in PBS did not rise as rapidly ("instantaneously") as those of the 20 complexes. This was also observed for FBP in acetonitrile (see preceding section), and may be attributed to an intramolecular vibrational relaxation. The situation would be different in the two complexes, where FBP is tightly bound to the protein, resulting in a reduced coupling of its vibrational modes. Moreover, such a 25 tight binding would imply a fast intermolecular vibrational relaxation which could accelerate the FBP intramolecular process. As a consequence, it would be too fast to be detected with the available time-resolution.

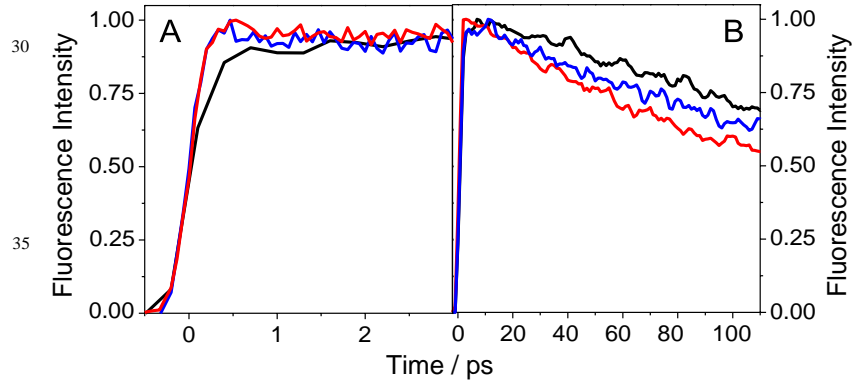

Figure 4. Normalised FU decays of ( $S$ )-FBP (black), $(S)$-FBP/HSA (red) 40 and $(R)$-FBP/HSA (blue) in PBS/air at $310 \mathrm{~nm}$. Note the different timescales used in $\mathrm{A}$ ) and $\mathrm{B}$ ).

Beyond a few picoseconds, the decays of the two FBP/HSA complexes became slightly more rapid than that of free FBP ${ }_{45}$ (Figure 4B). This behaviour can be explained in terms of a FBP dynamic quenching when bound to the protein, which is clearly configuration-dependent. The approximate characteristic decay times in the $0-120$ ps time window were 180 and 250 ps for $(S)$ FBP/HSA and $(R)-\mathrm{FBP} / \mathrm{HSA}$, respectively.

50 The dynamic quenching at $310 \mathrm{~nm}$ persisted and became even more marked at longer times, as illustrated by the TCSPC traces shown in Figure 5A. While the fluorescence decay of FBP was monoexponential with a lifetime of $0.78 \mathrm{~ns}$, that of HSA was more complex and can be described by an average lifetime $\langle\tau\rangle=$ $551.22 \mathrm{~ns}$. On the nanosecond timescale, the fluorescence decays of the two FBP/HSA complexes decayed much faster than free FBP.

This was more pronounced for the $(S)$ - than for the $(R)$ enantiomer $(\langle\tau\rangle=0.54 \mathrm{~ns} v s .0 .70 \mathrm{~ns}$, Table 2$)$.

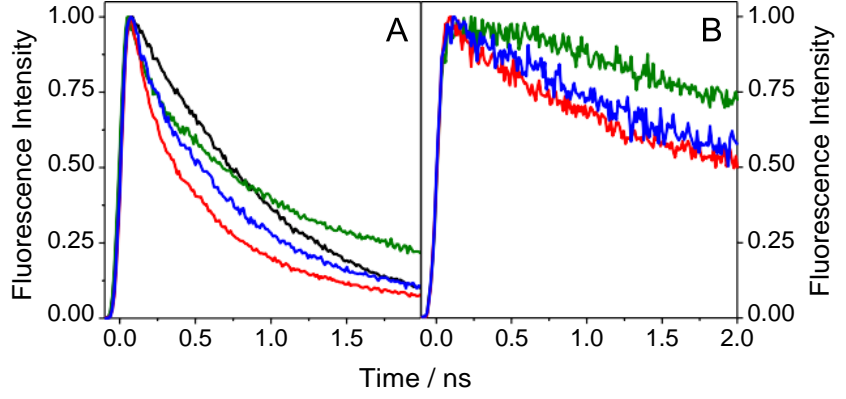

Figure 5. Normalised TCSPC decays of (S)-FBP (black), HSA (green), $60(S)$-FBP/HSA (red) and ( $R$ )-FBP/HSA (blue) in PBS at A) $\lambda_{\text {em }}=310 \mathrm{~nm}$, and B) $\lambda_{\mathrm{em}}=380 \mathrm{~nm}$.

At $380 \mathrm{~nm}$ (Figure 5B), where only HSA emits, the fluorescence lifetimes were shorter than that of HSA alone (Table 2). Again, there was a clear stereoselectivity in this process.

65 The HSA fluorescence is known to be highly nonexponential, ${ }^{36}$ but the actual characteristic decay times depend on both the excitation and the emission wavelengths, ranging from less than one to several nanoseconds. ${ }^{49-51}$ This observation has been explained by the heterogeneity of the Trp 70 microenvironment. ${ }^{49}$ Previous femtosecond studies on UV excited HSA showed that the dynamics of Trp within the protein are slower than in solution, ${ }^{52-54}$ in line with our observations.

Regarding the anisotropies recorded by FU, several interesting aspects can be noted (Figure 6A). For HSA, an initial value of 75 about 0.18 was observed, the same as for isolated Trp. Thus, the HSA fluorescence is dominated by Trp, and other residues contribute only marginally. The fluorescence anisotropy remained constant over the observed time window, in line with a slow rotational diffusion of the voluminous protein. In contrast, 80 for FBP, the initial fluorescence anisotropy at time zero $\left(r_{0}\right)$ was $0.36 \pm 0.02$ and decayed with a characteristic time of $65 \pm 6 \mathrm{ps}$ (in accordance with the rotational diffusion of FBP in water). However, in the presence of the protein, the $r_{0}$ value dropped to $0.29 \pm 0.02$ but remained practically constant during the first 85 hundreds of picoseconds. In principle, this can be explained by encapsulation of the drug within the more constrained protein microenvironment. Since overlapping fluorescence from both FBP and HSA was observed, the resulting anisotropy value (0.29) was between those of FBP (0.36) and HSA (0.18).

${ }_{90}$ Table 2. Kinetic parameters obtained from the fitting of the TCSPC decays upon excitation at $267 \mathrm{~nm}$ in PBS under air. Uncertainties are \pm 5 $\%$ unless otherwise stated.

\begin{tabular}{ccccc}
$\lambda_{\mathrm{em}}(\mathrm{nm})$ & Parameters $^{\mathrm{a}}$ & HSA & $(S)$-FBP/HSA & $(R)$-FBP/HSA \\
\cline { 3 - 5 } 310 & $\tau_{1}(\mathrm{~ns}) / \mathrm{p}_{1}(\%)$ & $0.16 / 5$ & $0.18 / 17$ & $0.25 / 9$ \\
& $\tau_{2}(\mathrm{~ns}) / \mathrm{p}_{2}(\%)$ & $1.00 / 36$ & $0.69 / 51$ & $0.72 / 60$ \\
& $\tau_{3}(\mathrm{~ns}) / \mathrm{p}_{3}(\%)$ & $4.36 / 59$ & $3.48 / 32$ & $3.51 / 31$ \\
& $\langle\tau\rangle(\mathrm{ns})$ & 1.22 & 0.54 & 0.70 \\
\hline \multirow{4}{*}{380} & $\tau_{1}(\mathrm{~ns}) / \mathrm{p}_{1}(\%)$ & $2.94 / 18$ & $0.36 / 3$ & $0.53 / 3$ \\
& $\tau_{2}(\mathrm{~ns}) / \mathrm{p}_{2}(\%)$ & $6.93 / 71$ & $2.59 / 25$ & $3.08 / 30$ \\
& $\tau_{3}(\mathrm{~ns}) / \mathrm{p}_{3}(\%)$ & $12.8 / 11$ & $7.68 / 72$ & $7.95 / 67$ \\
& $\langle\tau\rangle(\mathrm{ns})$ & 5.78 & 3.69 & 4.14 \\
\hline
\end{tabular}

${ }^{\text {a }}$ Obtained by a non-linear fitting/deconvolution procedure, using a three exponential function $F(t)=\Sigma a_{i} \exp \left(-t / \tau_{i}\right) ; p_{i}=100 a_{i} \tau_{i} /\left(a_{1} \tau_{1}+a_{2} \tau_{2}+a_{3} \tau_{3}\right)$; ${ }_{95}\langle\tau\rangle$ is the average lifetime, determined as $a_{1} \tau_{1}+a_{2} \tau_{2}+a_{3} \tau_{3}$. At $310 \mathrm{~nm}$, $\tau_{\mathrm{F}}(\mathrm{FBP})$ was $0.78 \mathrm{~ns}$. 
The fluorescence anisotropy decays recorded by TCSPC at $\lambda_{\mathrm{em}}$ $=310 \mathrm{~nm}$ (ns timescale) are shown in Figure 6B. In contrast with the ps kinetics recorded by FU, a chiral discrimination was observed. For both drug/protein systems, the $r_{0}$ value was $c a$. ${ }_{5} 0.28 \pm 0.02$ and decreased rapidly to a constant value within the first few nanoseconds. Monoexponential fitting gave a characteristic time of $0.44 \pm 0.03 \mathrm{~ns}$ for $(S)$-FBP/HSA and $0.62 \pm$ $0.07 \mathrm{~ns}$ for $(R)-\mathrm{FBP} / \mathrm{HSA}$. The difference in lifetimes can be related to the orientation of the drug within the protein, which

10 may restrict the degrees of freedom for conformational relaxation more effectively in the case of the $(R)$-enantiomer.

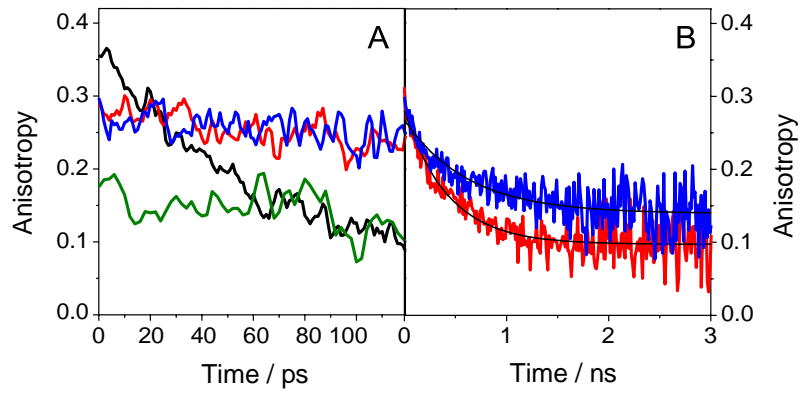

Figure 6. Fluorescence anisotropy decays of ( $S$ )-FBP (black), HSA (green), $(S)$-FBP/HSA (red) and $(R)$-FBP/HSA (blue) in PBS at $\lambda_{\text {em }}=310$ $15 \mathrm{~nm}$. A) FU and B) TCSPC (the best fit it is shown in black solid line).

Actually, it is well know that for a chromophore attached to a protein its motional freedom is restricted. Such restricted rotational diffusion is commonly described by the "wobbling-ina-cone" model $^{55}$

( ) $\left[\begin{array}{llll}( & ) & / & ] \\ \ddagger & ( & \end{array}\right]$

25 where $\theta_{\max }$ corresponds to the semicone angle defining the restricted motion of the chromophore. Using $r_{0}=0.28$ and $r_{\infty}=$ $0.10 / 0.14$ for $(S)$ - and $(R)$-FBP, values of $\theta_{\max }$ were calculated as $45^{\circ}$ and $38^{\circ}$, respectively. Therefore, $(R)$-FBP will cover a smaller solid angle during its rotational diffusion than the $(S)$ 30 enantiomer. This, together with the fact that its characteristic reorientational time is much slower indicates a more restricted conformation of the $(R)$-enantiomer within the protein.

\section{Conclusions}

The goal of the present work is to elucidate the interaction 35 between the two enantiomers of flurbiprofen and tryptophan. To this aim, we have compared the photophysical behaviour of the drug when covalently linked to Trp in model dyads with that of its non-covalent complex with human serum albumin.

A dramatic fluorescence quenching is observed in the dyads, 40 which display only a residual emission assigned to the Trp unit. According to the analysis of the FU decays, this quenching is dynamic $\left(\mathrm{k}>10^{10} \mathrm{~s}^{-1}\right)$ and stereoselective, with a higher rate constant for the $(R, S)$-diastereomer. The absence of ${ }^{1} \mathrm{FBP} *$ fluorescence has previously been attributed to energy transfer to
${ }_{45}$ Trp. ${ }^{38}$ While this explanation remains a possibility, it can neither be confirmed nor discarded by the present time-resolved experiments. At longer timescales, a slower stereoselective quenching $\left(\mathrm{k}>10^{9} \mathrm{~s}^{-1}\right)$ of the ${ }^{1} \operatorname{Trp} *$ fluorescence is also observed, together with exciplex formation.

50 Similar trends were observed in the drug/protein complexes, although the kinetics of the involved processes are slower. The fluorescence decay at $\lambda_{\mathrm{em}}=310 \mathrm{~nm}$ (FBP maximum) revealed a stereoselective dynamic quenching, both on the picosecond (FU) and nanosecond (TCSPC) timescales. This kinetic 55 stereodifferentiation was still evident at longer wavelengths (380 $\mathrm{nm}$ ), where only HSA is emitting. As in the dyads, the nature of this slower quenching can be attributed to a stereoselective exciplex formation and/or electron transfer.

Finally, the anisotropy at $310 \mathrm{~nm}$ recorded by TCSPC clearly 60 showed that the protein microenvironment plays a significant role in the conformational relaxation of FBP, which is more restricted in the case of the $(R)$-enantiomer. This stereoselectivity is possibly related to the modes of drug binding to the protein, a process of pharmacological relevance.

${ }_{65}$ Comparing the behaviour of the dyads with that of the complex, the same fundamental processes occur in the two systems, although on different timescales. The observed dynamic quenching rates are much lower in the latter, which can be understood in terms of the strong conformation dependence of the 70 involved processes.

It should be noted that stereoelectronic effects are quite sensitive to the vector approach of the interacting partners. This is because a critical factor in stereodifferentiation is the relative spatial arrangement of the reactive sites, which is strongly 75 influenced by the steric hindrance found in the approach trajectories. Hence, the limitation of the degrees of freedom imposed by the covalent linker in the dyads is not comparable to the restrictions associated with the non-covalent, supramolecular binding existing in the protein complexes. As a consequence, the 80 interest of the employed dyads as models is that they allow us to predict the interchromophoric excited state interactions and to assess the dynamic nature of quenching, as well as to anticipate the possibility of observing stereodifferentiation in the involved processes. The magnitude of the kinetic rate constants, as well as 85 the sign of stereodifferentiation, are expectedly difficult to reproduce, also because the dyads lack the tertiary structure of proteins, whose folding generates the binding sites for complexed ligands. However, this limitation does not diminish at all the value of the dyads as well-defined chemical models to interrogate

90 relevant interactions between photoactive drugs and the key amino acids present at the protein binding sites.

\section{Acknowledgements}

Financial support from the Spanish Government (Grants CTQ2010-14882 and CTQ2009-13699, JCI-2011-09926, BES95 2008-003314), the Generalitat Valenciana (Prometeo 2008/090) and from the Universitat Politècnica de València (PAID 05-11, Ref 2766) is gratefully acknowledged. 


\section{Notes and references}

${ }^{a}$ Departamento de Química/Instituto de Tecnología Química UPV-CSIC, Universitat Politècnica de València, 46022 Valencia, Spain. Fax: +34967879349; Tel: +34967877344; e-mail: mmiranda@qim.upv.es.

${ }_{5}$ CNRS, IRAMIS, SPAM, Francis Perrin Laboratoire, URA 2453, 91191

Gif-sur-Yvette, France. Fax: +330169087639; Tel: +330169084644; email: thomas.gustavsson@cea.fr

1 T. Peters, in All About Albumin - Biochemistry, Genetics, and Medical 10 Applications, Academic Press, San Diego, 1995, ch. 3, pp. 76-132.

2 D. C. Carter and J. X. Ho, Advances in Protein Chemistry, 1994, 45, 153-203.

3 U. Madsen, P. Kroogsgaard-Larsen and T. Liljefors, Textbook of Drug Design and Discovery, Taylor and Francis, Washington DC, 2002.

154 B. Zimmermann, C. Hahnefeld and F. W. Herberg, Targets, 2002, 1, 6673.

5 J. Rovensky and D. Micekova, Drug Exp. Clin. Res, 2000, 26, 19-24.

6 N. Bellamy, W. G. Bensen, P. M. Ford, S. H. Huang and J. Y. Lang, Clin. Invest. Med., 1992, 15, 427-433.

207 D. S. Muckle, Am. J. Med., 1986, 80, 76-80.

8 M. Vetrugno, A. Maino, G. M. Quaranta and L. Cardia, Clin. Ther., 2000, 22, 719-731.

9 H. A. Bae, K. W. Lee and Y. H. Lee, J. Mol. Catal. B-Enzym., 2006, 40, 24-29.

2510 S. Sagdinc and H. Pir, Spectrochim. Acta A, 2009, 73, 181-194.

11 T. Wybranowski, M. Cyrankiewicz, B. Ziomkowska and S.

Kruszewski, Biosystems, 2008, 94, 258-262.

12 Y. V. Il'ichev, J. L. Perry and J. D. Simon, J. Phys. Chem. B, 2002, 106, $452-459$.

3013 Y. V. Il'ichev, J. L. Perry and J. D. Simon, J. Phys. Chem. B, 2002, 106, $460-465$.

14 M. C. Jimenez, M. A. Miranda, R. Tormos and I. Vaya, Photochem. Photobiol. Sci., 2004, 3, 1038-1041.

15 I. Vaya, C. J. Bueno, M. C. Jimenez and M. A. Miranda, ${ }_{35}$ ChemMedChem, 2006, 1, 1015-1020.

16 N. Seedher and S. Bhatia, J. Pharm. Biomed. Anal., 2005, 39, $257-$ 262.

17 N. Seedher and S. Bhatia, Pharmacol. Res., 2006, 54, 77-84.

18 R. K. Nanda, N. Sarkar and R. Banerjee, J. Photochem. Photobiol. A, 40 2007, 192, 152-158.

19 Y. He, Y. W. Wang, L. F. Tang, H. Liu, W. Chen, Z. L. Zheng and G. L. Zou, J. Fluoresc., 2008, 18, 433-442.

20 B. Zhou, R. Li, Y. Zhang and Y. Liu, Photochem. Photobiol. Sci., 2008, 7, 453-459.

4521 I. Vaya, R. Pérez-Ruiz, V. Lhiaubet-Vallet, M. C. Jiménez and M. A. Miranda, Chem. Phys. Lett., 2010, 486, 147-153.

22 H. Vahedian-Movahed, M. R. Saberi and J. Chamani, J. Biomol. Struct. Dyn., 2011, 28, 483-502.

23 B. Hemmateenejad, M. Shamsipur, F. Samari, T. Khayamian, M. 50 Ebrahimi and Z. Rezaei, J. Pharm. Biomed. Anal., 2012, 67-68, 201-208.

24 U. Katrahalli, V. K. A. Kalalbandi and S. Jaldappagari, J. Pharm. Biomed. Anal., 2012, 59, 102-108.

25 M. El-Kemary, M. Gil and A. Douhal, J. Med. Chem., 2007, 50, 2896-2902.

${ }_{55} 26$ L. Tormo, J. A. Organero, B. Cohen, C. Martin, L. Santos and A. Douhal, J. Phys. Chem. B, 2008, 112, 13641-13647.

27 S. Tardioli, I. Lammers, J. H. Hooijschuur, F. Ariese, G. van der Zwan and C. Gooijer, J. Phys. Chem. B, 2012, 116, 7033-7039.

28 D. P. Zhong, A. Douhal and A. H. Zewail, Proc. Natl. Acad. Sci. 60 U.S.A., 2000, 97, 14056-14061.

29 A. Douhal, M. Sanz and L. Tormo, Proc. Natl. Acad. Sci. U.S.A., 2005, 102, 18807-18812.

30 B. Cohen, J. A. Organero, L. Santos, L. R. Padial and A. Douhal, J. Phys. Chem. B, 2010, 114, 14787-14795.

6531 M. Gil, Y. Wang and A. Douhal, J. Photochem. Photobiol. A, 2012, 234, 146-155.

32 Y. L. Wang, B. Cohen, L. Jicsinszky and A. Douhal, Langmuir, 2012, 28, 4363-4372.

33 P. G. Takla, S. G. Schulman and J. H. Perrin, J. Pharm. Biomed. 70 Anal., 1985, 3, 41-50.
34 I. Lammers, V. Lhiaubet-Vallet, M. C. Jiménez, F. Ariese, M. A. Miranda and C. Gooijer, Chirality, 2012, 24,840-846.

35 M. Amiri, K. Jankeje and J. R. Albani, J. Fluoresc., 2010, 20, 651656.

7536 J. R. Lakowicz, Principles of Fluorescence Spectroscopy, Plenum Press, New York, 2006, ch. 16, 17, pp .530-600.

37 I. Vaya, M. C. Jimenez and M. A. Miranda, J. Phys. Chem. B, 2007, 111, 9363-9371.

38 M. C. Jiménez, U. Pischel and M. A. Miranda, J. Photochem. 80 Photobiol. C, 2007, 8, 128-142.

39 S. Abad, U. Pischel and M. A. Miranda, J. Phys. Chem. A, 2005, 109, 2711-2717.

40 S. Abad, I. Vaya, M. C. Jimenez, U. Pischel and M. A. Miranda, ChemPhysChem, 2006, 7, 2175-2183.

8541 M. Montalti, A. Credi, L. Prodi and M. T. Gandolfi, Handbook of Photochemistry, CRC Press, Taylor and Francis Group, Boca Raton Fl., 2006.

42 D. Rehm and. A. Weller, Isr. J. Chem. 1970, 8, 259-271.

43 T. Gustavsson, A. Sharonov and D. Markovitsi, Chem. Phys. Lett., 90 2002, 351, 195-200.

44 F. A. Miannay, T. Gustavsson, A. Banyasz and D. Markovitsi, J. Phys. Chem. A, 2010, 114, 3256-3263.

45 D. Markovitsi, D. Onidas, F. Talbot, S. Marguet, T. Gustavsson and E. Lazzarotto, J. Photochem. Photobiol. A, 2006, 183, 1-8.

${ }_{95} 46$ P. Bonancía, I. Vayá, M. J. Climent, T. Gustavsson, D. Markovitsi, M. C. Jiménez and M. A. Miranda, J. Phys. Chem. A, 2012, 116, 88078814.

47 J. W. Petrich, M. C. Chang, D. B. McDonald and G. R. Fleming, J. Am. Chem. Soc., 1983, 105,3824-3832.

$10048 \mathrm{H}$. Lemmetyinen, N. Tkachenko, A. Efimov and M. Niemi, $J$. Porphyrins Phthalocyanines, 2009, 13, 1090-1097.

49 A. Siemiarczuk, C. E. Petersen, C. E. Ha, J. S. Yang and N. V. Bhagavan, Cell Biochem. Biophys., 2004, 40,115-122.

50 J. M. Beechem and L. Brand, Annu. Rev. Biochem., 1985, 54,43-71.

10551 A. Sarkar and S. C. Bhattacharya, J. Lumin., 2012, 132, 2612-2618.

52 W. Lu, J. Kim, W. Qiu and D. Zhong, Chem. Phys. Lett., 2004, 388, 120-126.

53 W. Qiu, L. Zhang, O. Okobiah, Y. Yang, L. Wang, D. Zhong and A. H. Zewail, J. Phys. Chem. B, 2006, 110,10540-10549.

11054 L. Zhang, Y.-T. Kao, W. Qiu, L. Wang and D. Zhong, J. Phys. Chem. $B, 2006,110,18097-18103$.

55 G. F. Schroder, U. Alexiev and H. Grubmuller, Biophys. J., 2005, 89, 3757-3770.

115 